\title{
A network meta-analysis on the efficacy of sixteen targeted drugs in combination with chemotherapy for treatment of advanced/metastatic colorectal cancer
}

\author{
Dan-Zeng Ba-Sang ${ }^{1}$, Zi-Wen Long ${ }^{2,3}$, Hao Teng ${ }^{1}$, Xu-Peng Zhao ${ }^{1}$, Jian Qiu ${ }^{1}$, Ming-Shan Li ${ }^{1}$ \\ ${ }^{1}$ Department of Oncology, Shigatse People's Hospital, Shigatse 857000, Tibet, P. R. China \\ ${ }^{2}$ Department of Gastric Cancer and Soft-Tissue Sarcoma Surgery, Fudan university Shanghai Cancer Center, Shanghai \\ 200032, P. R. China \\ ${ }^{3}$ Department of Oncology, Shanghai Medical College, Fudan University, Shanghai 200032, P. R. China
}

Correspondence to: Zi-Wen Long, email: longziwen576@163.com

Keywords: colorectal cancer, targeted drug, chemotherapy, randomized controlled trial, network meta-analysis

Received: May 06, 2016

Accepted: October 02, 2016

Published: October 31, 2016

\section{ABSTRACT}

Objective: A network meta-analysis was conducted comparing the short-term efficacies of 16 targeted drugs in combination with chemotherapy for treatment of advanced/metastatic colorectal cancer (CRC).

Results: Twenty-seven RCTs were ultimately incorporated into this network meta-analysis. Compared with chemotherapy alone, bevacizumab + chemotherapy, panitumumab + chemotherapy and conatumumab + chemotherapy had higher PR rate. Bevacizumab + chemotherapy, cetuximab + chemotherapy, panitumumab + chemotherapy, trebananib + chemotherapy and conatumumab + chemotherapy had higher ORR rate in comparison to chemotherapy alone. Furthermore, bevacizumab + chemotherapy had higher DCR rate than chemotherapy alone. The results of our cluster analysis showed that chemotherapy combined with bevacizumab, cetuximab, panitumumab, conatumumab, ganitumab, or brivanib + cetuximab had better efficacies for the treatment of advanced/metastatic CRC in comparison to chemotherapy alone.

Materials and Methods: Electronic databases were comprehensively searched for potential and related randomized controlled trials (RCTs). Direct and indirect evidence were incorporated for evaluation of stable disease (SD), progressive disease (PD), complete response (CR), partial response (PR), disease control rate (DCR) and overall response ratio (ORR) by calculating odds ratio (OR) and $95 \%$ confidence intervals (CI), and using the surface under the cumulative ranking curve (SUCRA).

Conclusions: These results indicated that bevacizumab + chemotherapy, panitumumab + chemotherapy, conatumumab + chemotherapy and brivanib + cetuximab + chemotherapy may have better efficacies for the treatment of advanced/ metastatic CRC.

\section{INTRODUCTION}

Colorectal Cancer (CRC) is a malignant disease of worldwide concern mainly affecting the elderly with a general onset age of 66 years old [1]. According to the World Cancer Report, there were 694,000 CRC deaths in 2012 [2]. The survival rate for CRC in a 5-year period increased from $51 \%$ to $65 \%$ due to developments in medical science and treatment methods, but fatal metastatic CRC remains is significant threat to public health [3]. Currently, surgical resection combined with chemotherapy is the frontline treatment for $\mathrm{CRC}$; however, chemotherapy remains flawed, as the desired therapeutic effects are achieved in only $30 \%$ of patients. This might be due to the fact that conventional chemotherapy drugs are toxic to both tumor and normal tissues, and therefore cause a variety of side effects, including neutropenia, anemia, stomach poisoning disease, hematopoietic disorders and poisoning stem cells [4]. The application of drugs specifically targeting tumor tissue would reduce the interference to normal tissues and decrease side effects [5]. 
Current targeted drugs can be roughly divided into the following categories: (1) drugs that inhibit VEGF-related factors to reduce angiopoiesis, including bevacizumab [6], cediranib [7], axitinib [8], and sorafenib [9]; (2) drugs that inhibit EGFR-related factors to control tumor cell proliferation and differentiation, including cetuximab [10], panitumumab [11], and gefitinib [12]; (3) drugs that inhibit angiopoietin, including sunitinib [13], celecoxib [14], and trebananib [15]; and (4) drugs that inhibit tumor proliferation though other relevant pathways, e.g. conatumumab causes apoptosis of cancer cells by targeting DR5 [16] and ganitumab inhibits tumor cell proliferation by inhibiting IGF signaling [17]. Combined treatment with anticancer drugs from different chemotherapy regimens is also commonly used. A previous study demonstrated that sunitinib-based combination was associated with more toxicity, and combination therapy with sunitinib plus mFOLFOX6 was not recommended in patients with metastatic colorectal cancer [18]. Moreover, the combination use of sorafenib in combination with mFOLFOX6 chemotherapy regimen was not preferable in metastatic colorectal cancer [19]. Despite the abundant literature submitted, no comprehensive literature regarding the optimal strategy on combined targeted drugs with chemotherapy in colorectal cancer was available.

The study of targeted drugs is based on clinical data from long-term evaluation of patients, for which the selection of methodology is an important factor affecting research conclusions [20]. Meta-analysis is a commonly used clinical analytical tool; with sufficient data its conclusions are normally reliable. However, traditional meta-analysis is often applied to paired comparison, in which case, the conclusions often less accurate [20]. Network meta-analysis can make up this deficiency, implementing a direct and indirect quantified comparison of different treatments with similar disease interventions and then selecting the optimal treatment [21]. This study integrates the research data of 16 targeted drugs combined with chemotherapy for the treatment of advanced/metastatic $\mathrm{CRC}$, attempting to uncover the best treatment options for advanced/metastatic CRC through network meta-analysis.

\section{RESULTS}

\section{Baseline characteristics of included study}

Our electronic database searches provided 2084 candidate studies. After reviewing the titles and abstracts, we excluded 513 studies as duplicates, 105 as letters or summaries, 76 as non-English studies, 557 as unrelated to advanced/metastatic CRC, and 567 as non-targeted drug studies. Upon further assessment of the remaining 266 articles, we excluded 109 studies of non-RCTs, 101 studies without data resources or incomplete documentation, 4 articles by the same author with the same content in a different document, 11 articles with incomplete documentation on shortterm efficacy, and 14 studies of non-fluorouracil-based chemotherapy. Eventually, 27 RCTs were found eligible for this network meta-analysis [18, 19, 22-46] and the PRISMA screening flow chart (see Figure 1). Together these 27 RCTs included 9031 patients with advanced/ metastatic CRC, including 2584 cases treated with chemotherapy alone (19 studies), 1936 cases treated with bevacizumab + chemotherapy (15 studies), 1598 cases treated with cetuximab + chemotherapy (7 studies), 632 cases treated with panitumumab + chemotherapy ( 3 studies), 482 cases treated with sunitinib + chemotherapy (2 studies), 853 cases treated with cediranib + chemotherapy ( 2 study), 19 cases treated with celecoxib + chemotherapy (1 study ) and 97 cases treated with sorafenib + chemotherapy (1 study), 133 cases treated with axitinib + chemotherapy (3 studies), 95 cases treated with trebananib + chemotherapy (1 study), 51 cases treated with conatumumab + chemotherapy (1 study), 52 cases treated with ganitumab + chemotherapy (1 study), 137 cases treated with panitumumab + bevacizumab + chemotherapy (1 study ), 376 cases treated with brivanib + cetuximab + chemotherapy (1 study), 82 cases treated with axitinib + bevacizumab + chemotherapy (2 studies), and 123 cases treated with cetuximab + bevacizumab + chemotherapy ( 1 study). The included studies were published between 2006 and 2015. Baseline characteristics of the included studies were summarized in Supplementary Table S1. Cochrane systematic bias for all included studies was shown in Figure 2.

\section{Pairwise meta-analysis}

We carried out direct paired comparisons for the shortterm efficacies of 16 treatments on advanced/metastatic CRC patients. Cetuximab + chemotherapy, sunitinib + chemotherapy, sorafenib + chemotherapy, panitumumab + bevacizumab + chemotherapy for advanced/metastatic CRC patients had lower SD rate than chemotherapy alone (all $P<0.05$ ). The PD rate of bevacizumab + chemotherapy for advanced/metastatic CRC patients was relatively lower than chemotherapy alone $(\mathrm{OR}=0.533$, $P=0.004)$. Bevacizumab + chemotherapy and cetuximab + chemotherapy had higher PR rate than chemotherapy alone (both $P<0.05$ ). In terms of ORR rate, bevacizumab + chemotherapy, cetuximab + chemotherapy, panitumumab + chemotherapy were better than chemotherapy alone (all $P<0.05)$. However, the $\mathrm{CR}$ and DCR rates of all targeted drugs combined with chemotherapy were not significantly different with chemotherapy alone (all $P>0.05$ ) (Supplementary Table S2).

\section{Forest plots for the efficacy of 16 targeted drugs combined with chemotherapy}

The relative relationships to direct and indirect short-term efficacy of the 16 targeted drugs combined with chemotherapy for advanced/metastatic CRC patients showed that: (1) The SD rates of cetuximab + 
chemotherapy and trebananib + chemotherapy were relatively lower than chemotherapy alone, while the SD rates of other targeted drugs combined with chemotherapy showed no statistically significant difference with chemotherapy alone. (2) The PD rates of bevacizumab + chemotherapy and brivanib + cetuximab + chemotherapy were relatively lower than that chemotherapy alone. No difference was also found between other targeted drugs combined with chemotherapy and chemotherapy alone. (3) The CR rates of the 16 targeted drugs combined with chemotherapy showed no obvious difference with that of chemotherapy alone. (4) The PR rates of bevacizumab + chemotherapy, panitumumab + chemotherapy, conatumumab + chemotherapy were higher than that of chemotherapy alone, while other targeted drugs combined with chemotherapy showed no difference with chemotherapy alone. (5) The ORR rates of bevacizumab + chemotherapy, cetuximab + chemotherapy, panitumumab + chemotherapy, trebananib + chemotherapy, and conatumumab + chemotherapy were higher than that of chemotherapy alone, while other targeted drugs combined with chemotherapy showed no significant difference with the efficacy of chemotherapy alone. (6) The DCR rates of bevacizumab + chemotherapy was better than that of chemotherapy alone, while the efficacy of other targeted drugs in combination with chemotherapy had no statistical difference when compared to the efficacy of chemotherapy (Figure 3, Supplementary Table S3).

\section{Evidence network of network meta-analysis}

A total of 17 kinds of treatment regimens were included in the current study (A: chemotherapy; $\mathrm{B}$ : bevacizumab + chemotherapy; $\mathrm{C}$ : celecoxib + chemotherapy; D: panitumumab + chemotherapy; E: sunitinib + chemotherapy; F: cediranib + chemotherapy; $\mathrm{H}$ : sorafenib + chemotherapy; I: axitinib + chemotherapy; $\mathrm{J}$ : trebananib + chemotherapy; K: conatumumab + chemotherapy; L: ganitumab + chemotherapy; M: gefitinib + chemotherapy; $\mathrm{N}$ : panitumumab + bevacizumab + chemotherapy; O: brivanib + cetuximab + chemotherapy; P: axitinib + bevacizumab + chemotherapy; Q: cetuximab + bevacizumab + chemotherapy). The network plot displays the short-term effects of the 17 treatment regimens on patients with advanced/metastatic CRC, and the data for every direct comparison of two treatment regimens are included in Figure 4.

\section{Inconsistency test of network meta-analysis}

We performed an inconsistency test on the 27 included studies which constituted 4 closed loops that formed a ring of research studies (5 studies of chemotherapy vs. bevacizumab + chemotherapy; 4 studies of chemotherapy vs. cetuximab + chemotherapy; 2 studies of bevacizumab + chemotherapy vs. cetuximab + chemotherapy; 5 studies of chemotherapy vs. bevacizumab + chemotherapy; 2 studies of chemotherapy + chemotherapy vs. panitumumab +

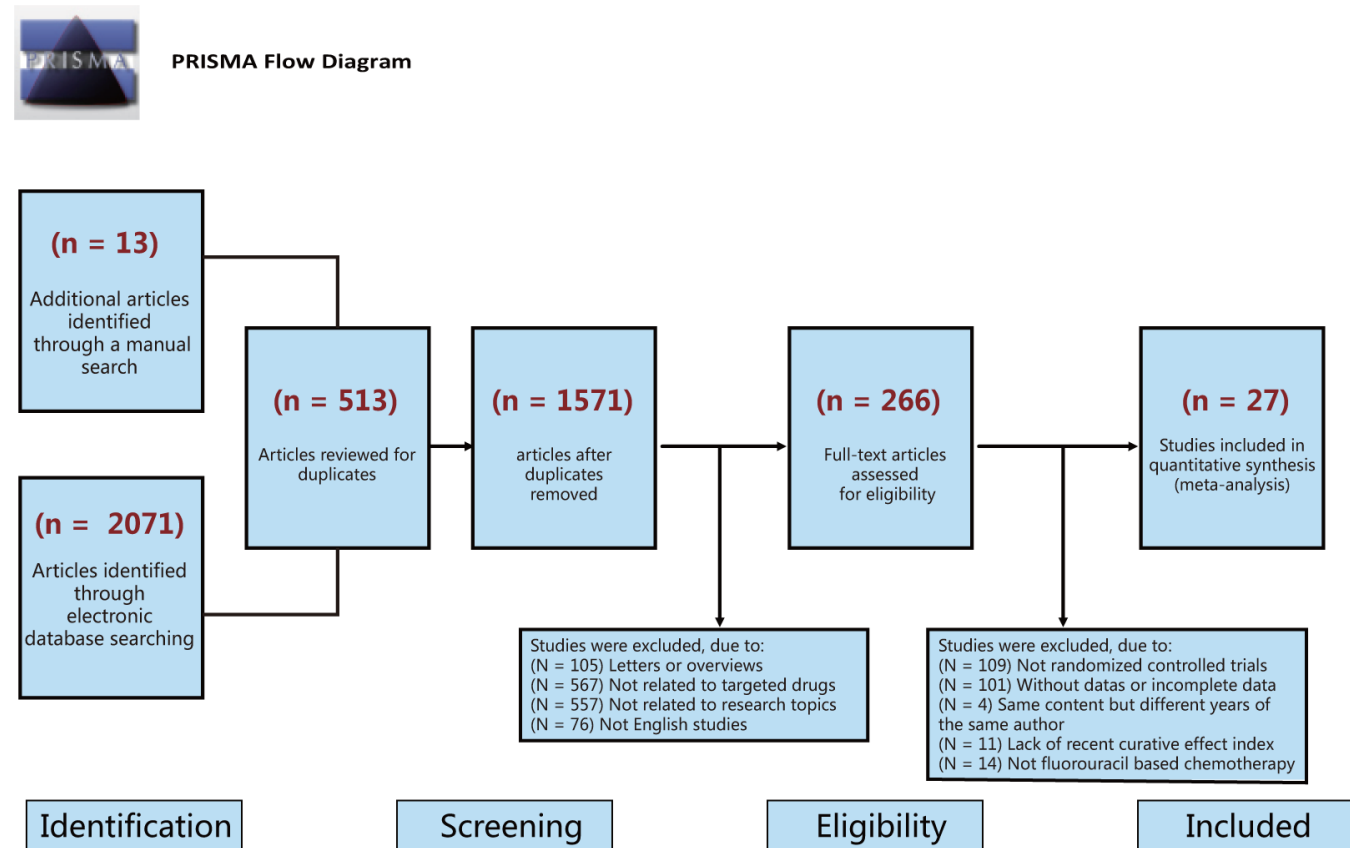

Figure 1: Flow chart showing literature search and study selection. Twenty-seven randomized controlled trials that met the inclusion criteria were included in this network meta-analysis. 
chemotherapy, 1 study of bevacizumab + chemotherapy vs. panitumumab + chemotherapy; 5 studies of chemotherapy vs. bevacizumab + chemotherapy; 1 study of chemotherapy vs. sunitinib + chemotherapy; 1 study of bevacizumab + chemotherapy vs. sunitinib + chemotherapy; 2 studies of bevacizumab + chemotherapy vs. axitinib + chemotherapy; 1 study of bevacizumab + chemotherapy vs. axitinib + bevacizumab + chemotherapy, 1 study of axitinib + chemotherapy vs. axitinib + bevacizumab + chemotherapy). The results showed no inconsistencies among the studies in terms of SD, PD, CR, PR, DCR, or ORR (all $P>0.05$ ) (Table 1). Therefore, the consistency model is applicable.

\section{Surface under the cumulative ranking curves (SUCRA)}

The SUCRA values of the 16 treatment regimens for advanced/metastatic CRC patients are summarized in Table 2. The SUCRA curves indicated that: (1) the combined therapies of cetuximab + bevacizumab + chemotherapy, sorafenib + chemotherapy, bevacizumab + chemotherapy, and axitinib + bevacizumab + chemotherapy had lower $\mathrm{SD}$ rates for the treatment of advanced/metastatic CRC treatment; the SUCRA values of which were 0.81 , $0.79,0.65$, and 0.65 , respectively; (2) the combined therapies of brivanib + cetuximab + chemotherapy, axitinib + bevacizumab + chemotherapy, bevacizumab + chemotherapy had lower PD rates for the treatment of advanced/metastatic CRC, the SUCRA values of which were $0.86,0.78$, and 0.72 , respectively; (3) the combined therapies of cetuximab + chemotherapy, brivanib + cetuximab + chemotherapy, and sorafenib + chemotherapy had higher $\mathrm{CR}$ rates for the treatment of advanced/metastatic CRC, the SUCRA values of which were 0.74, 0.69, and 0.66, respectively; (4) the combined therapies of conatumumab + chemotherapy, trebananib + chemotherapy, ganitumab + chemotherapy had higher PR rates for the treatment of advanced/metastatic CRC, the SUCRA values of which were $0.90,0.86$, and 0.80 , respectively; (5) the combined therapies of conatumumab + chemotherapy, trebananib + chemotherapy, and ganitumab + chemotherapy had higher ORR rates for the treatment of advanced/metastatic CRC, the SUCRA values of which were $0.91,0.88$, and 0.79 , respectively; and (6) the combined therapies of bevacizumab + chemotherapy, cetuximab + bevacizumab + chemotherapy, brivanib + cetuximab + chemotherapy had higher DCR rates for the treatment of advanced/metastatic CRC, the SUCRA value of which were $0.86,0.74$, and 0.73 , respectively.

\section{Cluster analysis}

Cluster analysis of SUCRA values based on $\mathrm{SD}$ vs. PD, SD vs. PR, SD vs. ORR, SD vs. DCR shows that the combined therapies of bevacizumab + chemotherapy, cetuximab + chemotherapy, panitumumab + chemotherapy, conatumumab + chemotherapy, ganitumab + chemotherapy, brivanib + cetuximab + chemotherapy were relatively good for the treatment of advanced/metastatic CRC. SUCRA value results of cluster analysis based on ORR vs. DCR showed that: the combined therapies of bevacizumab + chemotherapy, cetuximab + chemotherapy, panitumumab + chemotherapy, conatumumab + chemotherapy, ganitumab + chemotherapy and brivanib + cetuximab + chemotherapy were relatively good in efficacy for the treatment of advanced/metastatic CRC (Figure 5).

\section{DISCUSSION}

Integrating currently available data, current network meta-analysis of targeted drug therapy proved that the efficacies of the combined therapies were better than the efficacy of chemotherapy alone. Our results showed that the efficacy of bevacizumab + chemotherapy, sorafenib +

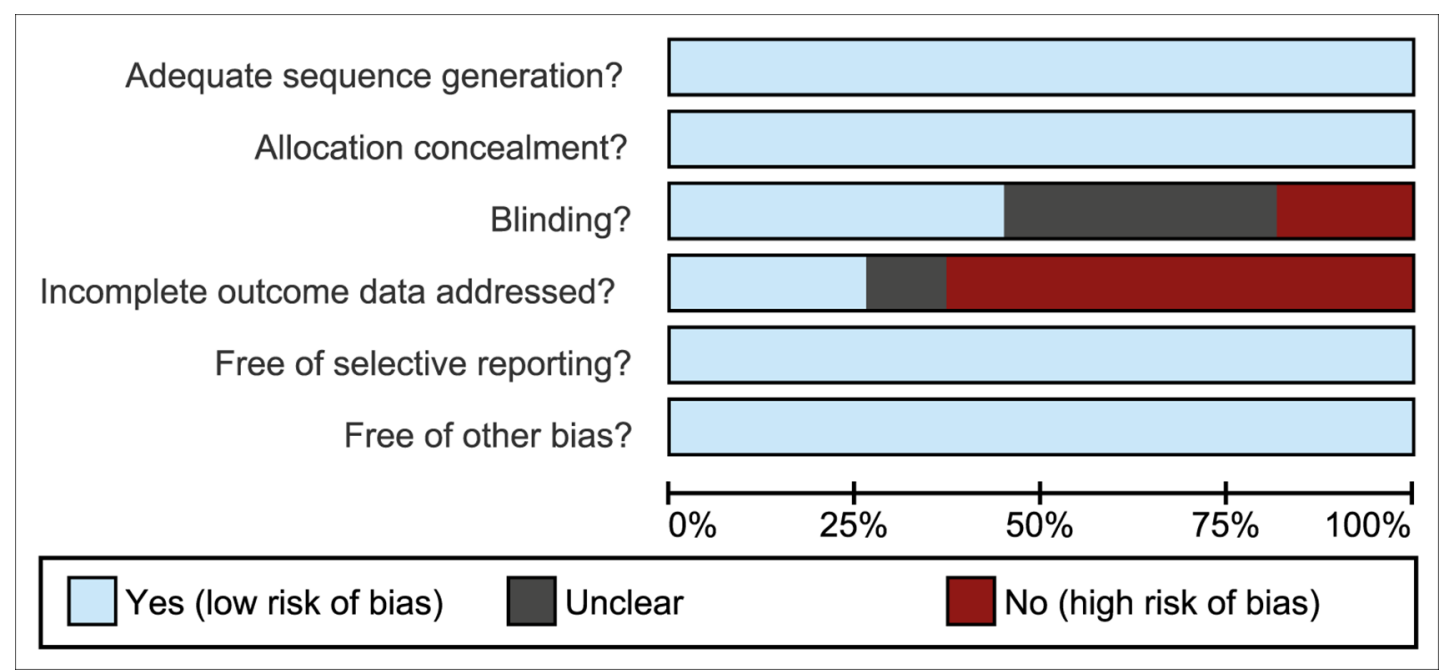

Figure 2: Quality assessment diagram of 27 included studies in this network meta-analysis. 
A

\section{Stable disease}

Comparison

$B$ vs $A$

$C$ vs $A$

$D$ vs $A$

E vs $A$

$F$ vs $A$

$G$ vs $A$

$H$ vs $A$

I vs $A$

$J$ vs $A$

$K$ vs $A$

L vs A

$M$ vs $A$

$\mathrm{N}$ vs $\mathrm{A}$

O vs A

$P$ vs $A$

$Q$ vs $A$

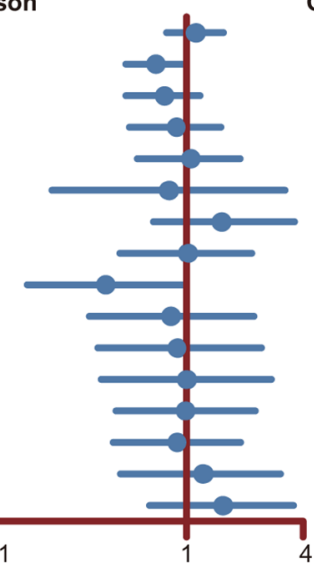

C

\section{Complete response}

\section{Comparison}

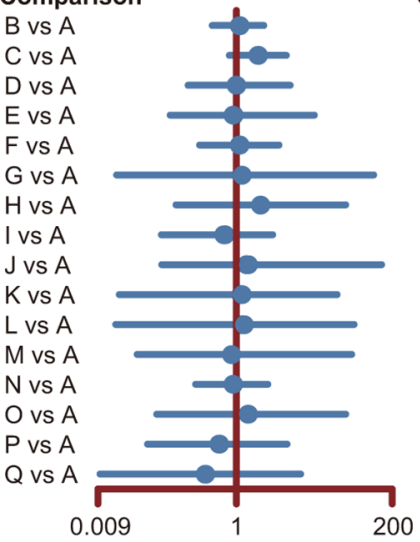

E

Overall respose rate

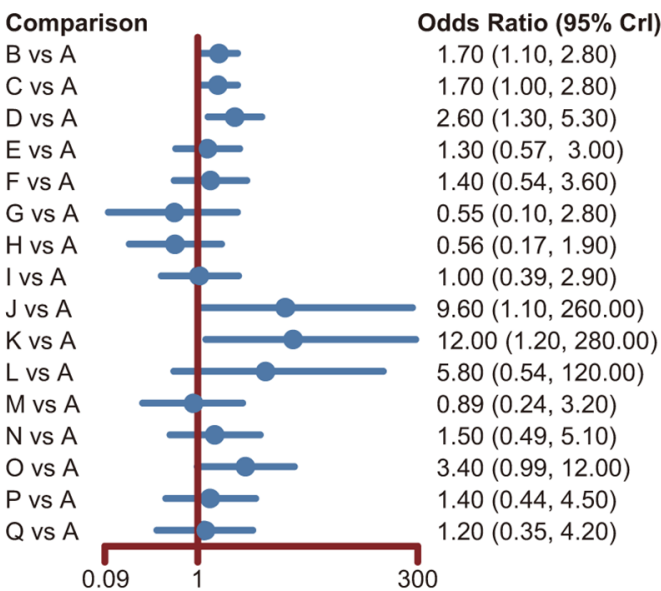

Odds Ratio (95\% Crl) $1.10(0.44,2.50)$

$2.10(0.79,5.50)$

$1.00(0.19,6.20)$

$0.93(0.11,14.00)$

$1.10(0.29,4.30)$

$2.20(0.13,41.00)$

$0.68(0.08,3.40)$

$1.40(0.08,140.00)$

$1.20(0.02,31.00)$

$1.20(0.02,55.00)$

$0.85(0.03,50.00)$

$0.92(0.25,2.90)$

$1.50(0.07,41.00)$

$0.56(0.05,5.60)$

$0.35(0.01,8.90)$
B

\section{Progressive disease}

Odds Ratio (95\% Crl)

$1.10(0.79,1.60)$

$0.69(0.49,0.99)$

$0.77(0.49,1.20)$

$0.89(0.51,1.50)$

$1.10(0.56,1.90)$

$0.81(0.20,3.20)$

$1.50(0.67,3.60)$

$1.00(0.45,2.20)$

$0.38(0.15,0.98)$

$0.83(0.31,2.20)$

$0.90(0.35,2.40)$

$1.00(0.36,2.70)$

$0.99(0.43,2.30)$

$0.89(0.42,1.90)$

$1.20(0.46,3.10)$

$1.50(0.64,3.60)$

Comparison

$C$ vs $A$

$D$ vs $A$

$E$ vs $A$

$F$ vs $A$

$G$ vs $A$

$H$ vs $A$

I vs $A$

$J$ vs $A$

$K$ vs $A$

L vs $A$

$M$ vs $A$

$N$ vs $A$

$O$ vs $A$

$P$ vs $A$

$Q$ vs $A$
$B$ vs $A$

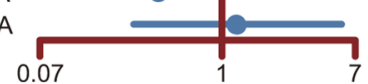

D

\section{Partial response}

$1.20(0.02,110.00)$

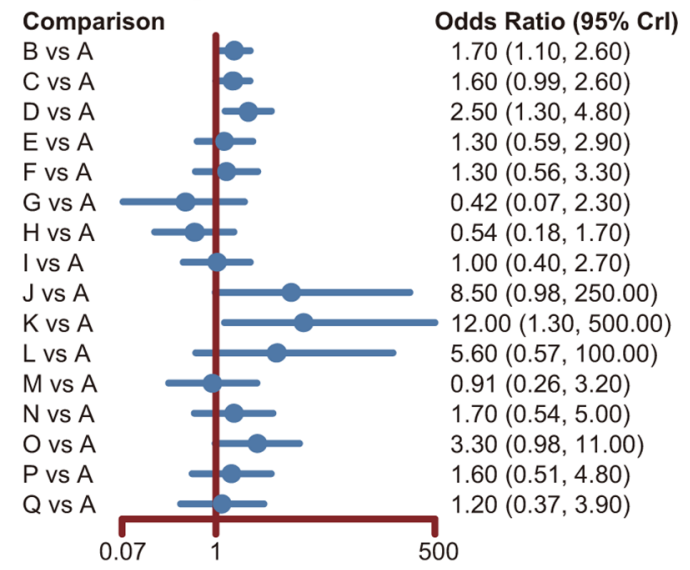

F

\section{Disease control rate}

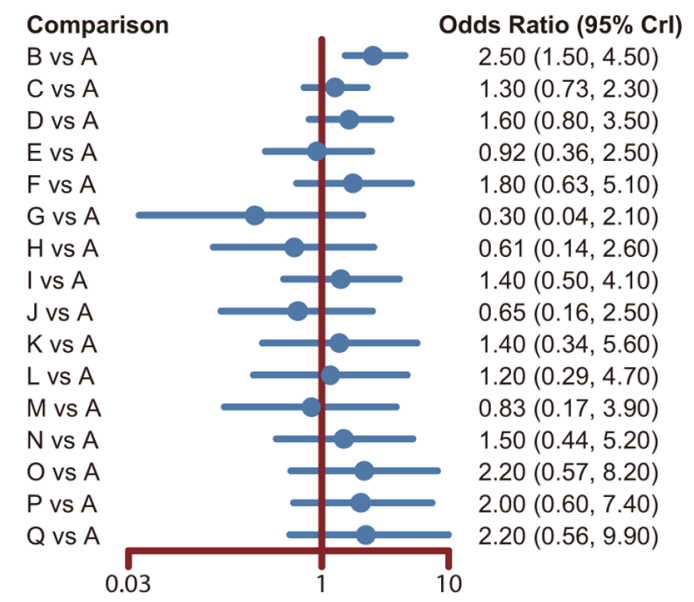

Odds Ratio (95\% Crl)

$0.49(0.29,0.78)$

$0.75(0.44,1.10)$

$0.83(0.35,2.10)$

$0.60(0.25,1.40)$

$0.69(0.07,5.60)$

$0.73(0.16,3.10)$

$0.51(0.16,1.40)$

$1.60(0.51,5.20)$

$0.88(0.25,3.40)$

$0.62(0.16,2.40)$

$1.50(0.38,6.40)$

$0.53(0.20,1.40)$

$0.34(0.11,0.88)$

$1.20(0.28,5.70)$
$0.67(0.37,1.20)$

$0.40(0.09,1.50)$

Figure 3: Forest plots for the efficacy of 16 targeted drugs combined with chemotherapy in the treatment of advanced/ metastatic colorectal cancer. The black solid lines represent the confidence intervals for summary odds ratios for each comparison. (A: chemotherapy; B: bevacizumab + chemotherapy; C: cetuximab + chemotherapy; D: panitumumab + chemotherapy; E: sunitinib + chemotherapy; F: cediranib + chemotherapy; G: celecoxib + chemotherapy; H: sorafenib + chemotherapy; I: axitinib + chemotherapy; $\mathrm{J}$ : trebananib + chemotherapy; K: conatumumab + chemotherapy; L: ganitumab + chemotherapy; M: gefitinib + chemotherapy; N: panitumumab + bevacizumab + chemotherapy; O: brivanib + cetuximab + chemotherapy; P: axitinib + bevacizumab + chemotherapy; Q: cetuximab + bevacizumab + chemotherapy). 
chemotherapy, panitumumab + chemotherapy, trebananib + chemotherapy and conatumumab + chemotherapy were more prominent among all the combined chemotherapies. Evidence supported that monoclonal antibody-based treatment of cancer has been proved as one of the most successful therapeutic strategies for both hematologic malignancies and solid tumors $[47,48]$. Several antibodies were included as targeted drugs in current study, including bevacizumab and conatumumab. Bevacizumab is an antiVEGF monoclonal antibody, which has a good efficacy and can prolong the survival time of advanced/metastatic CRC patients [23-24]. Sorafenib is a multi-targeted tyrosine kinase, which can control angiogenesis by inhibiting the VEGF receptor, effective for the treatment of tumors [29]. Besides, the combined therapy of trebananib with anti-VEGF drugs, such as sorafenib, bevacizumab or motesanib, presented with apparently less toxicity and more effective in inhibiting the proliferation of tumors cells $[49,50]$. Conatumumab is a monoclonal antibody which targets at DR5 and thus mediates the apoptosis of tumor cells with low toxicity to normal cells [19, 27, 51]. Conatumumab is a relevant factor that can cause apoptosis of the tumor cells and can be applied with combination of 5-fluorouracil, leucovorin and irinotecan. The combination of these agents can reduce the toxic effects of the body and thus distinctively mediate the apoptosis of tumor cells [52]. Consistent with these findings, the results of this study showed that the efficacies of bevacizumab + chemotherapy, panitumumab + chemotherapy, conatumumab + chemotherapy and trebananib + chemotherapy were relatively more significant than the efficacy of chemotherapy alone. Panitumumab is a complex which inhibits the expression of downstream molecules indirectly by binding to EGFR, so as to inhibit the proliferation and differentiation of tumor cells [24]. Trebananib is a peptide hybrid protein, which can deter the formation of tumors by interfering with the binding of angiopoietin-1 and angiopoietin-2 to Tie2 receptor [28].

Additionally, our study found that the efficacy of brivanib + celecoxib + chemotherapy is distinctively better than the efficacies of cetuximab + chemotherapy and sorafenib + chemotherapy. A perspective study has demonstrated that both brivanib and cetuximab inhibit the VEGF signaling pathway, so as to cooperate with each other in inhibiting tumor cell-induced angiogenesis $[29,30]$. Greening et al. also pointed out that the efficacy of brivanib and cetuximab was significantly better than the efficacy of cetuximab and cetuximab combined with irinotecan [53]. However, no direct report proves that the efficacy of combined brivanib and cetuximab therapy is better than the efficacy of Sorafenib therapy. In addition, the combined therapy of brivanib and cetuximab has been reported to produce a certain toxicity which has a negative impact on the overall survival of patients [28], which may explain why the efficiency of combined therapy of brivanib and cetuximab was inferior to that of trebananib. However, our results revealed that the efficacy of brivanib + celecoxib + chemotherapy is distinctively better than the efficacy of trebananib + chemotherapy. But further research is needed to discover the advantages and disadvantages of both therapies. From pharmacodynamic perspective, the advantages of the combined therapy of brivanib and cetuximab may occur because it not only inhibits VEGF-induced angiogenesis of tumor cells, but also inhibits EGFR signaling pathways in parallel to further inhibit the proliferation of tumor cells [54].

Further analysis of the results of SUCRA revealed that the efficacies of trebananib + chemotherapy, conatumumab + chemotherapy, and ganitumab + chemotherapy are relatively higher in regard to $\mathrm{PR}$ and ORR. This may due to the fact that the included documents and data are relatively limited, which may result in a deviation of the results.

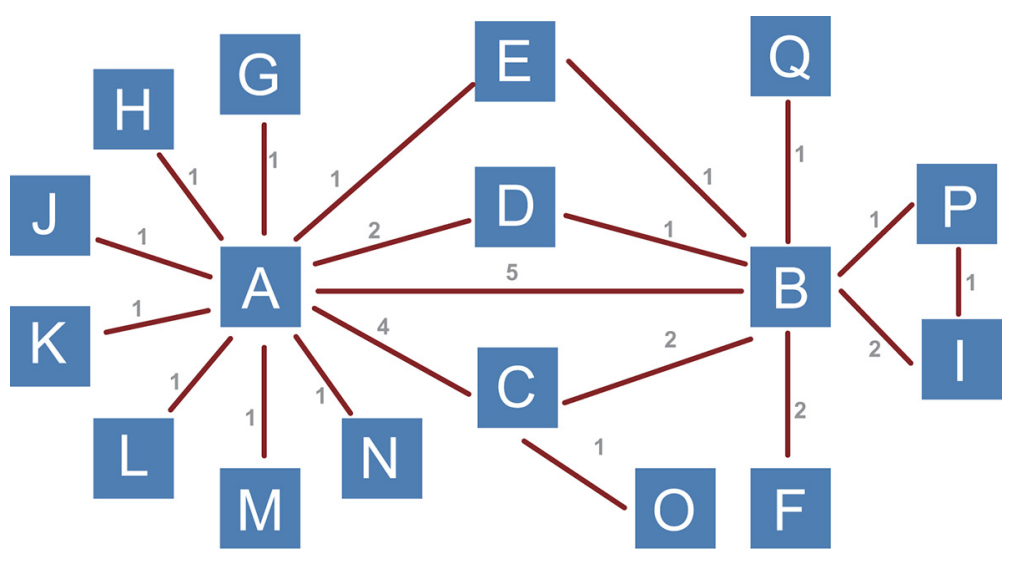

Figure 4: Evidence network plot of 17 treatment regimens which were included in this network meta-analysis. (A: chemotherapy; $\mathrm{B}$ : bevacizumab + chemotherapy; C: cetuximab + chemotherapy; D: panitumumab + chemotherapy; E: sunitinib + chemotherapy; F: cediranib + chemotherapy; G: celecoxib + chemotherapy; H: sorafenib + chemotherapy; I: axitinib + chemotherapy; J: trebananib + chemotherapy; K: conatumumab + chemotherapy; L: ganitumab + chemotherapy; M: gefitinib + chemotherapy; N: panitumumab + bevacizumab + chemotherapy; O: brivanib + cetuximab + chemotherapy; P: axitinib + bevacizumab + chemotherapy; Q: cetuximab + bevacizumab + chemotherapy). 
Table 1: OR values and $P$ values of direct and indirect pairwise comparisons of seventeen treatment modalities under six endpoint outcomes

\begin{tabular}{|c|c|c|c|c|c|c|c|c|c|c|c|c|c|c|c|c|c|c|}
\hline \multirow{2}{*}{$\begin{array}{c}\text { Pairwise } \\
\text { comparisons }\end{array}$} & \multicolumn{6}{|c|}{ Direct OR values } & \multicolumn{6}{|c|}{ Indirect $O R$ values } & \multicolumn{6}{|c|}{$P$ values } \\
\hline & SD & PD & CR & PR & ORR & DCR & SD & PD & CR & PR & ORR & DCR & SD & PD & CR & PR & ORR & DCR \\
\hline A VS B & -0.017 & -0.730 & 0.405 & 0.603 & 0.690 & 0.982 & 0.275 & -0.432 & -0.501 & 0.355 & 0.313 & 0.774 & 0.334 & 0.488 & 0.268 & 0.556 & 0.389 & 0.681 \\
\hline A VS C & -0.362 & -0.187 & 0.334 & 0.526 & 0.549 & 0.250 & -0.366 & -0.562 & 1.385 & 0.297 & 0.432 & 0.185 & 0.993 & 0.444 & 0.246 & 0.653 & 0.828 & 0.915 \\
\hline A VS D & -0.171 & -0.336 & 0.006 & 0.798 & 0.802 & 0.386 & -0.608 & -0.534 & 0.136 & 1.227 & 1.327 & 0.785 & 0.356 & 0.741 & 0.942 & 0.538 & 0.472 & 0.595 \\
\hline A VS E & -0.032 & -0.278 & -0.010 & -0.051 & -0.063 & -0.172 & -0.319 & 0.553 & 0.124 & 0.682 & 0.723 & 0.070 & 0.577 & 0.501 & 0.948 & 0.300 & 0.296 & 0.795 \\
\hline B VS C & -0.474 & 0.136 & 1.022 & -0.157 & -0.087 & -0.696 & -0.471 & 0.511 & -0.029 & 0.072 & 0.030 & -0.631 & 0.993 & 0.444 & 0.246 & 0.653 & 0.829 & 0.915 \\
\hline B VS D & -0.668 & 0.100 & -0.001 & 0.682 & 0.734 & -0.160 & -0.231 & 0.298 & -0.130 & 0.253 & 0.209 & -0.560 & 0.356 & 0.741 & 0.942 & 0.538 & 0.472 & 0.595 \\
\hline B VS E & -0.400 & 1.109 & -0.011 & 0.116 & 0.115 & -0.843 & -0.113 & 0.278 & -0.145 & -0.617 & -0.671 & -1.085 & 0.576 & 0.501 & 0.948 & 0.300 & 0.296 & 0.795 \\
\hline B VS F & -0.057 & 0.157 & 0.023 & -0.215 & -0.217 & -0.350 & 0.202 & 1.124 & -0.516 & -1.099 & -1.219 & -1.131 & 0.998 & 0.994 & 0.999 & 0.993 & 0.991 & 0.994 \\
\hline B VS I & 0.032 & 0.189 & -0.376 & -0.384 & -0.414 & -0.376 & -0.611 & -0.537 & -0.693 & -0.803 & -0.870 & -1.425 & 0.463 & 0.558 & 0.881 & 0.674 & 0.657 & 0.313 \\
\hline B VS P & -0.167 & -0.511 & -0.668 & -0.213 & -0.400 & -0.672 & 0.475 & 0.215 & -0.352 & 0.206 & 0.056 & 0.376 & 0.464 & 0.558 & 0.881 & 0.674 & 0.657 & 0.313 \\
\hline B VS Q & 0.327 & 0.849 & -0.693 & -0.349 & -0.380 & -0.117 & -0.613 & -0.827 & -0.754 & -0.210 & -0.298 & -1.186 & 0.994 & 0.993 & 1.000 & 0.999 & 1.000 & 0.994 \\
\hline C VS O & 0.258 & -0.802 & -0.005 & 0.701 & 0.701 & 0.524 & 1.164 & 2.184 & -4.997 & -4.003 & -4.214 & -2.238 & 0.994 & 0.987 & 0.992 & 0.972 & 0.970 & 0.985 \\
\hline I VS P & 0.443 & 0.026 & 0.025 & 0.590 & 0.470 & 0.752 & -0.199 & -0.700 & -0.292 & 0.171 & 0.014 & -0.296 & 0.463 & 0.558 & 0.881 & 0.674 & 0.657 & 0.313 \\
\hline
\end{tabular}

Notes: $\mathrm{SD}=$ stable disease; $\mathrm{PD}=$ progressive disease; $\mathrm{CR}=$ complete response; $\mathrm{PR}=$ partial response; $\mathrm{ORR}=$ overall response rate; $\mathrm{DCR}=$ disease control rate; $\mathrm{ORR}=\mathrm{CR}+\mathrm{PR} ; \mathrm{DCR}=\mathrm{SD}+\mathrm{CR}+\mathrm{PR} ;$ $\mathrm{OR}=$ odd ratios; $\mathrm{A}=$ chemotherapy; $\mathrm{B}=$ bevacizumab+chemotherapy; $\mathrm{C}=$ cetuximab+chemotherapy; $\mathrm{D}=$ panitumumab+chemotherapy; $\mathrm{E}=$ sunitinib + chemotherapy; $\mathrm{F}=$ cediranib+chemotherapy; $\mathrm{I}=$ axitinib+chemotherapy; $\mathrm{O}=$ brivanib+cetuximab+chemotherapy; $\mathrm{P}=$ axitinib+bevacizumab+chemotherapy; $\mathrm{Q}=$ cetuximab+bevacizumab+chemotherapy.

Table 2: The results of surface under the cumulative ranking curve of 16 targeted drugs

\begin{tabular}{crllllll}
\hline Code & \multicolumn{1}{c}{ Treatment regimen } & SD & PD & CR & PR & ORR & DCR \\
\hline A & Chemotherapy & 0.55 & 0.27 & 0.46 & 0.26 & 0.22 & 0.34 \\
B & Bevacizumab + Chemotherapy & 0.65 & 0.72 & 0.53 & 0.56 & 0.57 & 0.86 \\
C & Cetuximab + Chemotherapy & 0.24 & 0.46 & 0.74 & 0.53 & 0.55 & 0.49 \\
D & Panitumumab + Chemotherapy & 0.33 & 0.52 & 0.53 & 0.72 & 0.72 & 0.62 \\
E & Sunitinib + Chemotherapy & 0.43 & 0.42 & 0.51 & 0.41 & 0.40 & 0.34 \\
F & Cediranib + Chemotherapy & 0.59 & 0.57 & 0.51 & 0.43 & 0.44 & 0.66 \\
G & Celecoxib + Chemotherapy & 0.43 & 0.55 & 0.51 & 0.10 & 0.16 & 0.13 \\
H & Sorafenib + Chemotherapy & 0.79 & 0.50 & 0.66 & 0.12 & 0.13 & 0.24 \\
I & Axitinib + Chemotherapy & 0.55 & 0.65 & 0.36 & 0.30 & 0.30 & 0.54 \\
J & Trebananib + Chemotherapy & 0.06 & 0.14 & 0.50 & 0.86 & 0.88 & 0.22 \\
K & Conatumumab + Chemotherapy & 0.42 & 0.38 & 0.47 & 0.90 & 0.91 & 0.54 \\
L & Ganitumab + Chemotherapy & 0.49 & 0.59 & 0.50 & 0.80 & 0.79 & 0.47 \\
M & Gefitinib + Chemotherapy & 0.53 & 0.18 & 0.54 & 0.28 & 0.28 & 0.31 \\
N & Panitumumab + Bevacizumab + Chemotherapy & 0.53 & 0.66 & 0.45 & 0.54 & 0.49 & 0.57 \\
O & Brivanib + Cetuximab + Chemotherapy & 0.46 & 0.86 & 0.69 & 0.77 & 0.77 & 0.73 \\
P & Axitinib + Bevacizumab + Chemotherapy & 0.65 & 0.78 & 0.32 & 0.51 & 0.44 & 0.71 \\
Q & Cetuximab + Bevacizumab + Chemotherapy & 0.81 & 0.27 & 0.25 & 0.37 & 0.38 & 0.74 \\
\hline
\end{tabular}

Notes: $\mathrm{SD}=$ stable disease; $\mathrm{PD}=$ progressive disease $\mathrm{CR}=$ complete response; $\mathrm{PR}=$ partial response; $\mathrm{ORR}=$ overall response rate; $\mathrm{DCR}=$ disease control rate; $\mathrm{ORR}=\mathrm{CR}+\mathrm{PR} ; \mathrm{DCR}=\mathrm{SD}+\mathrm{CR}+\mathrm{PR}$.

Another reason may be the exclusion criteria based on the analysis of pharmacological effects. As mentioned earlier, trebananib is effective in inhibiting the angiogenesisrelated pathway in tumor cells [49, 50]. Ganitumab inhibits the IGF signaling pathway by binding to IGF1R and thus inhibits proliferation of tumor cells. Although limited data on the efficacy of ganitumab combined with other drugs exists, clinical research shows that combined therapy of panitumumab and rilotumumab is much more efficacious than ganitumab therapy alone [55]. Thus, the actual efficacy of ganitumab therapy requires further study.
Due to the limited references and data, this study has integrated the current number of chemotherapy drugs which are compared and analyzed without yielding obvious conclusions on the ideal drugs for the progression or metastasis of advanced/metastatic CRC. Deviation occurs in network meta-analysis when the data are insufficient for the evaluation [56, 57]. Correction of this deviation relies on the optimization of the new algorithm and more clinical and basic research data for further correction and investigation. In summary, the results of this network meta-analysis suggest that bevacizumab + chemotherapy, 
panitumumab + chemotherapy, conatumumab + chemotherapy and brivanib + cetuximab + chemotherapy may have better efficacy for the treatment of advanced/ metastatic CRC, which provide evidence toward further development of more effective treatment of advanced/ metastatic CRC and inspiration for further study.

\section{MATERIALS AND METHODS}

\section{Search strategy}

PubMed, Embase, Cochrane central register of controlled trials (CENTRAL), and other English language databases were searched from the inception of each database through September 2015. Searches were conducted using the keywords and combined words: colorectal neoplasms, colorectal tumor, colorectal cancer, colorectal carcinomas, bevacizumab, cetuximab, panitumumab, sunitinib, cediranib, celecoxib, sorafenib, axitinib, trebananib, conatumumab, ganitumab, gefitinib, randomized controlled trial, and chemotherapy.

\section{Inclusion and exclusion criteria}

The inclusion criteria were: (1) study design: randomized controlled trial (RCT); (2) study subjects: advanced/metastatic CRC patients; (3) treatment regimens: targeted drugs combined with chemotherapy; (4) end outcomes: stable disease (SD), progressive disease (PD), complete response (CR), partial response (PR), disease control rate (DCR), and overall response ratio (ORR). The exclusion criteria were: (1) non-English reference; (2) non-RCTs; (3) duplicated publications from the same author with different interventions; (4) review and letters; (5) non-human research subjects; (6) documents with insufficient end outcomes; (7) non-fluorouracil-based chemotherapy study.

\section{Data extraction}

Two reviewers extracted data from the enrolled studies using a specifically designed form. Additionally, a third reviewer was consulted if agreement could not be reached between these two reviewers. Two or more researchers reviewed the RCTs according to Cochrane risk of bias assessment tools [58], which includes six domains: adequate sequence generation, allocation concealment, blinding, incomplete outcome data addressed, free of selective reporting, and free of other biases.

\section{Statistical analysis}

Traditional pairwise meta-analyses were performed to directly compared the short-term efficacies (CR, PR, ORR, SD, PD and DCR) of 16 targeted drugs (Bevacizumab, Cetuximab, Panitumumab, Sunitinib, Cediranib, Celecoxib, Sorafenib, Axitinib, Trebananib, Conatumumab, Ganitumab, Gefitinib, Panitumumab plus Bevacizumab, Brivanib plus Cetuximab, Axitinib plus Bevacizumab, Cetuximab plus Bevacizumab) in

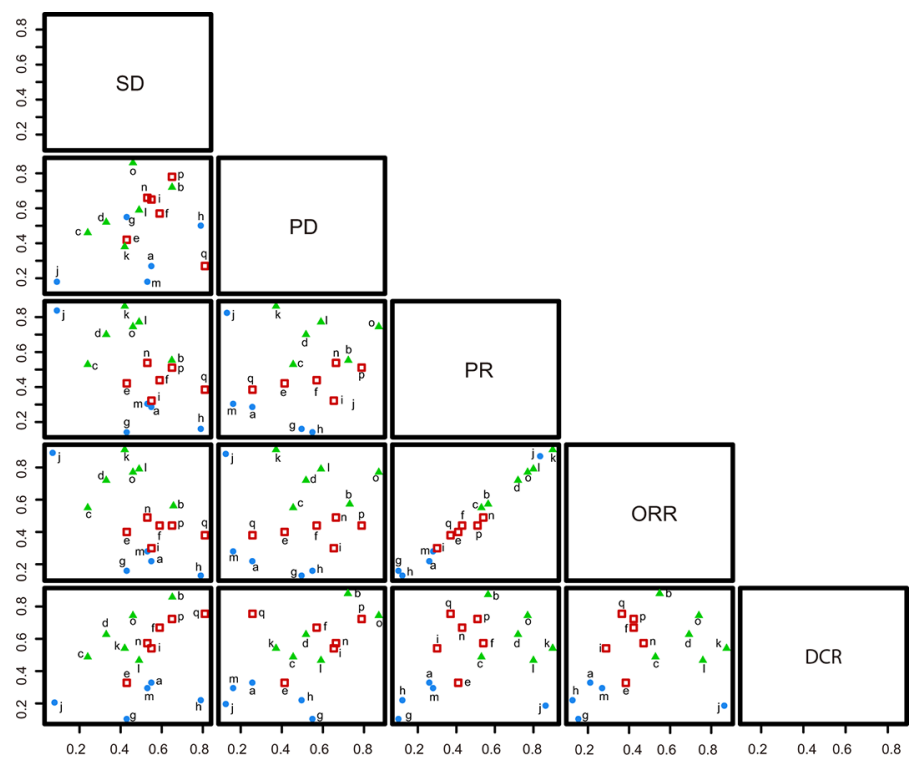

Figure 5: Clustered ranking plots based on SUCRA values of the SD, PD, PR, ORR and DCR rates of 17 treatment regimens in the treatment of advanced/metastatic colorectal cancer. (a: chemotherapy; b: bevacizumab + chemotherapy; c: cetuximab + chemotherapy; d: panitumumab + chemotherapy; e: sunitinib + chemotherapy; f: cediranib + chemotherapy; g: celecoxib + chemotherapy; h: sorafenib + chemotherapy; $\mathrm{i}=$ axitinib + chemotherapy $\mathrm{j}=$ trebananib + chemotherapy; $\mathrm{k}=$ conatumumab + chemotherapy $;=$ ganitumab + chemotherapy; $\mathrm{m}$ = gefitinib + chemotherapy; $\mathrm{n}=$ panitumumab + bevacizumab + chemotherapy; $\mathrm{o}=$ brivanib + cetuximab + chemotherapy; $\mathrm{p}$ $=$ axitinib + bevacizumab + chemotherapy; $\mathrm{q}=$ cetuximab + bevacizumab + chemotherapy $)$. 
combination with chemotherapy for the treatment of advanced/metastatic CRC. Bayesian network meta-analyses were performed to indirectly compare $\mathrm{CR}, \mathrm{PR}, \mathrm{ORR}, \mathrm{SD}$, $\mathrm{PD}$ and DCR of 16 targeted drugs in combination with chemotherapy for the treatment of advanced/metastatic CRC. Fixed-effect and random-effects model proposed by Woods and co-workers was applied. The node-splitting plot statistic was used to assess the extent of inconsistency, and ontology consistent model was applied if $P>0.05$. We checked and confirmed convergence and lack of auto correlation after four chains and a 20,000-simulation burnin phase. Direct probability statements were derived from an additional 50,000-simulation phase [59]. The surface under the cumulative ranking curve (SUCRA) was used to evaluate the efficacies of 16 targeted drugs in combination with chemotherapy for the treatment of advanced/ metastatic CRC [60]. The plot of a network of interventions is a visual representation of the evidence base and offers a concise description of its characteristics according to the SUCRA values, the larger the SUCRA value, the better the rank of the intervention [61]. The network meta-analyses were performed using Stata 13.1 (Stata Corp, College Station, TX, USA) software and R (V.3.1.2) package gemtc (V.0.6), 1314 along with the Markov Chain Monte Carlo engine Open BUGS (V.3.4.0).

\section{ACKNOWLEDGMENTS AND FUNDING}

We would like to acknowledge the helpful comments on this paper received from our reviewers.

\section{CONFLICTS OF INTEREST}

None declared.

\section{REFERENCES}

1. DeSantis CE, Lin CC, Mariotto AB, Siegel RL, Stein KD, Kramer JL, Alteri R, Robbins AS, Jemal A. Cancer treatment and survivorship statistics, 2014. CA. 2014; 64:252-271.

2. Esin E, Yalcin S. Maintenance strategy in metastatic colorectal cancer: A systematic review. Cancer Treat Rev. 2016; 42:82-90.

3. Ward E, DeSantis C, Robbins A, Kohler B, Jemal A. Childhood and adolescent cancer statistics, 2014. CA. 2014; 64:83-103.

4. Ades S. Adjuvant chemotherapy for colon cancer in the elderly: moving from evidence to practice. Oncology (Williston Park). 2009; 23:162-167.

5. Samant RS, Shevde LA. Recent advances in anti-angiogenic therapy of cancer. Oncotarget. 2011; 2:122-134. doi: 10.18632/oncotarget.234.

6. Gao H, Xue J, Zhou L, Lan J, He J, Na F, Yang L, Deng L, Lu Y. Bevacizumab radiosensitizes non-small cell lung cancer xenografts by inhibiting DNA double-strand break repair in endothelial cells. Cancer Lett. 2015; 365:79-88.

7. Decio A, Cesca M, Bizzaro F, Porcu L, Bettolini R, Ubezio P, Taraboletti G, Belotti D, Giavazzi R. Cediranib combined with chemotherapy reduces tumor dissemination and prolongs the survival of mice bearing patient-derived ovarian cancer xenografts with different responsiveness to cisplatin. Clin Exp Metastasis. 2015; 32:647-658.

8. Gunnarsson O, Pfanzelter NR, Cohen RB, Keefe SM. Evaluating the safety and efficacy of axitinib in the treatment of advanced renal cell carcinoma. Cancer Manag Res. 2015; 7:65-73.

9. White PT, Cohen MS. The discovery and development of sorafenib for the treatment of thyroid cancer. Expert Opin Drug Discov. 2015; 10:427-439.

10. Abdel-Rahman O, Fouad M. Correlation of cetuximabinduced skin rash and outcomes of solid tumor patients treated with cetuximab: a systematic review and metaanalysis. Crit Rev Oncol Hematol. 2015; 93:127-135.

11. Pettigrew M, Kavan P, Surprenant L, Lim HJ. Comparative net cost impact of the utilization of panitumumab versus cetuximab for the treatment of patients with metastatic colorectal cancer in Canada. J Med Econ. 2016; 19:135-147.

12. Dhillon S. Gefitinib: a review of its use in adults with advanced non-small cell lung cancer. Targeted oncology. 2015; 10:153-170.

13. Diaz R, Nguewa PA, Redrado M, Manrique I, Calvo A. Sunitinib reduces tumor hypoxia and angiogenesis, and radiosensitizes prostate cancer stem-like cells. Target Oncol. 2015; 75:1137-1149.

14. Reckamp KL, Koczywas M, Cristea MC, Dowell JE, Wang HJ, Gardner BK, Milne GL, Figlin RA, Fishbein MC, Elashoff RM, Dubinett SM. Randomized phase 2 trial of erlotinib in combination with high-dose celecoxib or placebo in patients with advanced non-small cell lung cancer. Cancer. 2015; 121:3298-3306.

15. Wu B, Melara R, Rasmussen E, Zhou L, Wong $T$, Kitahara C, Ma M, Lu J, Gamelin E, Pickett CA, Sun YN. Pharmacokinetic drug-drug interaction assessment of peptibody trebananib in combination with chemotherapies. Cancer Chemother Pharmacol. 2015; 76:243-250.

16. Tabernero J, Chawla SP, Kindler H, Reckamp K, Chiorean EG, Azad NS, Lockhart AC, Hsu CP, Baker NF, Galimi F, Beltran P, Baselga J. Anticancer activity of the type I insulin-like growth factor receptor antagonist, ganitumab, in combination with the death receptor 5 agonist, conatumumab. Target Oncol. 2015; 10:65-76.

17. Beltran PJ, Calzone FJ, Mitchell P, Chung YA, Cajulis E, Moody G, Belmontes B, Li CM, Vonderfecht S, Velculescu VE, Yang G, Qi J, Slamon DJ, et al. Ganitumab (AMG 479) inhibits IGF-II-dependent ovarian cancer growth and potentiates platinum-based chemotherapy. Clin Cancer Res. 2014; 20:2947-2958.

18. Hecht JR, Mitchell EP, Yoshino T, Welslau M, Lin X, Chow Maneval E, Paolini J, Lechuga MJ, Kretzschmar A. 
5-Fluorouracil, leucovorin, and oxaliplatin (mFOLFOX6) plus sunitinib or bevacizumab as first-line treatment for metastatic colorectal cancer: a randomized Phase IIb study. Cancer Manag Res. 2015; 7:165-173.

19. Tabernero J, Garcia-Carbonero R, Cassidy J, Sobrero A, Van Cutsem E, Kohne CH, Tejpar S, Gladkov O, Davidenko I, Salazar R, Vladimirova L, Cheporov S, Burdaeva O, et al. Sorafenib in combination with oxaliplatin, leucovorin, and fluorouracil (modified FOLFOX6) as first-line treatment of metastatic colorectal cancer: the RESPECT trial. Clin Cancer Res. 2013; 19:2541-2550.

20. Greco T, Zangrillo A, Biondi-Zoccai G, Landoni G. Metaanalysis: pitfalls and hints. Heart, lung and vessels. 2013; 5:219-225.

21. Greco T, Landoni G, Biondi-Zoccai G, D'Ascenzo F, Zangrillo A. A Bayesian network meta-analysis for binary outcome: how to do it. Stat Methods Med Res. 2013.

22. Hecht JR, Cohn A, Dakhil S, Saleh M, Piperdi B, ClineBurkhardt M, Tian Y, Go WY. SPIRITT: A randomized, multicenter, phase II study of panitumumab with FOLFIRI and bevacizumab with FOLFIRI as second-line treatment in patients with unresectable wild type KRAS metastatic colorectal cancer. Clin Colorectal Cancer. 2015; pp. 72-80.

23. Xie S, Han G, Fan Z, He L, Xu W, Qin Z. Safety and efficacy of second-line treatment with folinic acid, 5-fluorouracil and irinotecan (FOLFIRI) in combination of panitumumab and bevacizumab for patients with metastatic colorectal cancer. Med Oncol (Northwood, London, England). 2014; 31:35.

24. Peeters M, Price TJ, Cervantes A, Sobrero AF, Ducreux M, Hotko Y, Andre T, Chan E, Lordick F, Punt CJ, Strickland AH, Wilson G, Ciuleanu TE, et al. Final results from a randomized phase 3 study of FOLFIRI $\{+/-\}$ panitumumab for second-line treatment of metastatic colorectal cancer. Ann Oncol. 2014; 25:107-116.

25. Heinemann V, von Weikersthal LF, Decker T, Kiani A, Vehling-Kaiser U, Al-Batran SE, Heintges T, Lerchenmuller C, Kahl C, Seipelt G, Kullmann F, Stauch M, Scheithauer W, et al. FOLFIRI plus cetuximab versus FOLFIRI plus bevacizumab as first-line treatment for patients with metastatic colorectal cancer (FIRE-3): a randomised, open-label, phase 3 trial. Lancet Oncol. 2014; 15:1065-1075.

26. Cao R, Zhang S, Ma D, Hu L. A multi-center randomized phase II clinical study of bevacizumab plus irinotecan, 5-fluorouracil, and leucovorin (FOLFIRI) compared with FOLFIRI alone as second-line treatment for Chinese patients with metastatic colorectal cancer. Med Oncol (Northwood, London, England). 2014; pp. 1-5.

27. Ye LC, Liu TS, Ren L, Wei Y, Zhu DX, Zai SY, Ye QH, Yu Y, Xu B, Qin XY, Xu J. Randomized controlled trial of cetuximab plus chemotherapy for patients with KRAS wildtype unresectable colorectal liver-limited metastases. J Clin Oncol. 2013; 31:1931-1938.
38. Siu LL, Shapiro JD, Jonker DJ, Karapetis CS, Zalcberg JR, Simes J, Couture F, Moore MJ, Price TJ, Siddiqui J, Nott LM, Charpentier D, Liauw W, et al. Phase III randomized, placebo-controlled study of cetuximab plus brivanib alaninate versus cetuximab plus placebo in patients with metastatic, chemotherapy-refractory, wild-type K-RAS colorectal carcinoma: the NCIC Clinical Trials Group and AGITG CO.20 Trial. J Clin Oncol. 2013; 31:2477-2484.

29. Peeters M, Strickland AH, Lichinitser M, Suresh AV, Manikhas G, Shapiro J, Rogowski W, Huang X, Wu B, Warner D, Jain R, Tebbutt NC. A randomised, double-blind, placebo-controlled phase 2 study of trebananib (AMG 386) in combination with FOLFIRI in patients with previously treated metastatic colorectal carcinoma. Br J Cancer. 2013; 108:503-511.

30. Infante JR, Reid TR, Cohn AL, Edenfield WJ, Cescon TP, Hamm JT, Malik IA, Rado TA, McGee PJ, Richards DA, Tarazi J, Rosbrook B, Kim S, et al. Axitinib and/or bevacizumab with modified FOLFOX-6 as first-line therapy for metastatic colorectal cancer: a randomized phase 2 study. Cancer. 2013; 119:2555-2563.

31. Cunningham D, Wong RP, D’Haens G, Douillard JY, Robertson J, Stone AM, Van Cutsem E. Cediranib with mFOLFOX6 vs bevacizumab with mFOLFOX6 in previously treated metastatic colorectal cancer. Br J Cancer. 2013; 108:493-502.

32. Cohn AL, Tabernero J, Maurel J, Nowara E, Sastre J, Chuah BY, Kopp MV, Sakaeva DD, Mitchell EP, Dubey S, Suzuki S, Hei YJ, Galimi F, et al. A randomized, placebocontrolled phase 2 study of ganitumab or conatumumab in combination with FOLFIRI for second-line treatment of mutant KRAS metastatic colorectal cancer. Ann Oncol. 2013; pp. 1777-1785.

33. Carrato A, Swieboda-Sadlej A, Staszewska-Skurczynska M, Lim R, Roman L, Shparyk Y, Bondarenko I, Jonker DJ, Sun Y, De la Cruz JA, Williams JA, Korytowsky B, Christensen JG, et al. Fluorouracil, leucovorin, and irinotecan plus either sunitinib or placebo in metastatic colorectal cancer: a randomized, phase III trial. J Clin Oncol. 2013; 31:1341-1347.

34. Bendell JC, Tournigand C, Swieboda-Sadlej A, Barone C, Wainberg ZA, Kim JG, Pericay C, Pastorelli D, Tarazi J, Rosbrook B, Bloom J, Ricart AD, Kim S, et al. Axitinib or bevacizumab plus FOLFIRI or modified FOLFOX-6 after failure of first-line therapy for metastatic colorectal cancer: a randomized phase II study. Clin Colorectal Cancer. 2013; 12:239-247.

35. Stintzing S, Fischer von Weikersthal L, Decker T, VehlingKaiser U, Jager E, Heintges T, Stoll C, Giessen C, Modest DP, Neumann J, Jung A, Kirchner T, Scheithauer W, et al. FOLFIRI plus cetuximab versus FOLFIRI plus bevacizumab as first-line treatment for patients with metastatic colorectal cancer-subgroup analysis of patients with KRAS: mutated tumours in the randomised German AIO study KRK-0306. Ann Oncol. 2012; 23:1693-1699. 
36. Schmoll HJ, Cunningham D, Sobrero A, Karapetis CS, Rougier P, Koski SL, Kocakova I, Bondarenko I, Bodoky G, Mainwaring P, Salazar R, Barker P, Mookerjee B, et al. Cediranib with mFOLFOX6 versus bevacizumab with mFOLFOX6 as first-line treatment for patients with advanced colorectal cancer: a double-blind, randomized phase III study (HORIZON III). J Clin Oncol. 2012; 30:3588-3595.

37. Saltz L, Badarinath S, Dakhil S, Bienvenu B, Harker WG, Birchfield G, Tokaz LK, Barrera D, Conkling PR, O'Rourke MA, Richards DA, Reidy D, Solit D, et al. Phase III trial of cetuximab, bevacizumab, and 5-fluorouracil/ leucovorin vs. FOLFOX-bevacizumab in colorectal cancer. Clin Colorectal Cancer. 2012; 11:101-111.

38. Madajewicz S, Waterhouse DM, Ritch PS, Khan MQ, Higby DJ, Leichman CG, Malik SK, Hentschel P, Gill JF, Zhao L, Nicol SJ. Multicenter, randomized phase II trial of bevacizumab plus folinic acid, fluorouracil, gemcitabine (FFG) versus bevacizumab plus folinic acid, fluorouracil, oxaliplatin (FOLFOX4) as first-line therapy for patients with advanced colorectal cancer. Invest New Drugs. 2012; 30:772-778.

39. Matsusaka S, Suenaga M, Mishima Y, Takagi K, Terui Y, Mizunuma N, Hatake K. Circulating endothelial cells predict for response to bevacizumab-based chemotherapy in metastatic colorectal cancer. Cancer Chemother Pharmacol. 2011; pp. 763-768.

40. Guan ZZ, Xu JM, Luo RC, Feng FY, Wang LW, Shen L, Yu SY, Ba Y, Liang J, Wang D, Qin SK, Wang JJ, He J, et al. Efficacy and safety of bevacizumab plus chemotherapy in Chinese patients with metastatic colorectal cancer: a randomized phase III ARTIST trial. Chin J Cancer. 2011; 30:682-689.

41. Bokemeyer C, Bondarenko I, Hartmann JT, de Braud F, Schuch G, Zubel A, Celik I, Schlichting M, Koralewski P. Efficacy according to biomarker status of cetuximab plus FOLFOX-4 as first-line treatment for metastatic colorectal cancer: the OPUS study. Ann Oncol. 2011; 22:1535-1546.

42. Stathopoulos GP, Batziou C, Trafalis D, Koutantos J, Batzios S, Stathopoulos J, Legakis J, Armakolas A. Treatment of colorectal cancer with and without bevacizumab: a phase III study. Oncology. 2010; 78:376-381.

43. Van Cutsem E, Kohne CH, Hitre E, Zaluski J, Chang Chien CR, Makhson A, D'Haens G, Pinter T, Lim R, Bodoky G, Roh JK, Folprecht G, Ruff P, et al. Cetuximab and chemotherapy as initial treatment for metastatic colorectal cancer. N Engl J Med. 2009; 360:1408-1417.

44. Santoro A, Comandone A, Rimassa L, Granetti C, Lorusso V, Oliva C, Ronzoni M, Siena S, Zuradelli M, Mari E, Pressiani T, Carnaghi C. A phase II randomized multicenter trial of gefitinib plus FOLFIRI and FOLFIRI alone in patients with metastatic colorectal cancer. Ann Oncol. 2008; 19:1888-1893.

45. Kohne CH, De Greve J, Hartmann JT, Lang I, Vergauwe P, Becker K, Braumann D, Joosens E, Muller L, Janssens J,
Bokemeyer C, Reimer P, Link H, et al. Irinotecan combined with infusional 5-fluorouracil/folinic acid or capecitabine plus celecoxib or placebo in the first-line treatment of patients with metastatic colorectal cancer. EORTC study 40015. Ann Oncol. 2008; 19:920-926.

46. Maiello E, Giuliani F, Gebbia V, Di Renzo N, Pezzella G, Romito S, Mallamaci R, Lopez M, Colucci G. FOLFIRI with or without celecoxib in advanced colorectal cancer: a randomized phase II study of the Gruppo Oncologico dell'Italia Meridionale (GOIM). Ann Oncol. 2006; 17:vii55-59.

47. Lee CM, Tannock IF. The distribution of the therapeutic monoclonal antibodies cetuximab and trastuzumab within solid tumors. BMC Cancer. 2010; 10:255.

48. Scott A M AJP, Wolchok J D. Monoclonal antibodies in cancer therapy. Cancer Immunity Archive. 2012; 12:14.

49. Hong DS, Kurzrock R, Mulay M, Rasmussen E, Wu BM, Bass MB, Zhong ZD, Friberg G, Rosen LS. A phase 1b, open-label study of trebananib plus bevacizumab or motesanib in patients with solid tumours. Oncotarget. 2014; 5:11154-11167. doi: 10.18632/oncotarget.2568.

50. Hong DS, Gordon MS, Samlowski WE, Kurzrock R, Tannir N, Friedland D, Mendelson DS, Vogelzang NJ, Rasmussen E, Wu BM, Bass MB, Zhong ZD, Friberg G, et al. A phase I, open-label study of trebananib combined with sorafenib or sunitinib in patients with advanced renal cell carcinoma. Clin Genitourin Cancer. 2014; 12:167-177 e162.

51. Cao R, Zhang S, Ma D, Hu L. A multi-center randomized phase II clinical study of bevacizumab plus irinotecan, 5-fluorouracil, and leucovorin (FOLFIRI) compared with FOLFIRI alone as second-line treatment for Chinese patients with metastatic colorectal cancer. Med Oncol (Northwood, London, England). 2015; 32:325.

52. Cohn AL, Tabernero J, Maurel J, Nowara E, Sastre J, Chuah BY, Kopp MV, Sakaeva DD, Mitchell EP, Dubey S, Suzuki S, Hei YJ, Galimi F, et al. A randomized, placebocontrolled phase 2 study of ganitumab or conatumumab in combination with FOLFIRI for second-line treatment of mutant KRAS metastatic colorectal cancer. Ann Oncol. 2013; 24:1777-1785.

53. Cunningham D, Wong RP, D'Haens G, Douillard JY, Robertson J, Stone AM, Van Cutsem E and group HIs. Cediranib with mFOLFOX6 vs bevacizumab with mFOLFOX6 in previously treated metastatic colorectal cancer. Br J Cancer. 2013; 108:493-502.

54. Greening DW, Lee ST, Ji H, Simpson RJ, Rigopoulos A, Murone C, Fang C, Gong S, O'Keefe G, Scott AM. Molecular profiling of cetuximab and bevacizumab treatment of colorectal tumours reveals perturbations in metabolic and hypoxic response pathways. Oncotarget. 2015; 6:38166-38180. doi: 10.18632/oncotarget.6241.

55. Van Cutsem E, Eng C, Nowara E, Swieboda-Sadlej A, Tebbutt NC, Mitchell E, Davidenko I, Stephenson J, Elez E, Prenen H, Deng H, Tang R, McCaffery I, et al. 
Randomized phase Ib/II trial of rilotumumab or ganitumab with panitumumab versus panitumumab alone in patients with wild-type KRAS metastatic colorectal cancer. Clin Cancer Res. 2014; 20:4240-4250.

56. Jansen JP. Network meta-analysis of individual and aggregate level data. Res Synth Methods. 2012; 3:177-190.

57. Saramago P, Sutton AJ, Cooper NJ, Manca A. Mixed treatment comparisons using aggregate and individual participant level data. Stat Med. 2012; 31:3516-3536.

58. Higgins JP, Altman DG, Gotzsche PC, Juni P, Moher D, Oxman AD, Savovic J, Schulz KF, Weeks L, Sterne JA, Cochrane Bias Methods G, Cochrane Statistical Methods G. The Cochrane Collaboration's tool for assessing risk of bias in randomised trials. Bmj. 2011; 343:d5928.
59. Tu YK, Needleman I, Chambrone L, Lu HK, Faggion CM, Jr. A Bayesian network meta-analysis on comparisons of enamel matrix derivatives, guided tissue regeneration and their combination therapies. J Clin Periodontol. 2012; 39:303-314.

60. Salanti G, Ades AE, Ioannidis JP. Graphical methods and numerical summaries for presenting results from multipletreatment meta-analysis: an overview and tutorial. J Clin Epidemiol. 2011; 64:163-171.

61. Salanti G. Indirect and mixed-treatment comparison, network, or multiple-treatments meta-analysis: many names, many benefits, many concerns for the next generation evidence synthesis tool. Res Synth Methods. 2012; 3:80-97. 